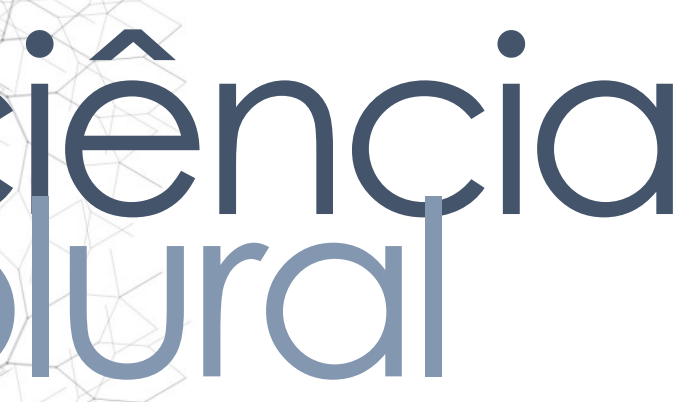

\title{
FATORES DESENCADEANTES DO ESTRESSE LABORAL NA EMERGÊNCIA MÉDICA: UMA REVISÃO INTEGRATIVA
}

Factors relating to workers 'stress in medical emergency: $a$ integrative review

Cecília Mirelle Almeida Honorato | Graduada em Medicina pela Universidade Federal do Rio Grande do Norte (UFRN) |

E-mail: cmirelle@hotmail.com

Flávia Christiane de Azevedo Machado |Odontóloga. Doutora em Saúde Coletiva pela Universidade Federal do Rio Grande do Norte (UFRN).

Docente do Departamento de Saúde Coletiva da UFRN |

E-mail: flavitamachado@yahoo.com.br

Autor responsável pela correspondência

Cecília Mirelle Almeida Honorato || E-mail: cmirelle@hotmail.com 


\section{RESUMO}

Introdução: Os serviços de emergência médica exigem do profissional da saúde uma pronta resposta às demandas de cada paciente. Nesse contexto, o estresse laboral pode ser extremamente destrutivo não só para o trabalhador, mas também para o serviço de saúde prestado. Objetivo: Assim, este estudo tem como objetivo analisar os fatores desencadeantes do estresse laboral em profissionais de saúde que trabalham em serviços de emergência médica. Metodologia: Para isso, foi realizada uma revisão integrativa de literatura, tendo como fonte as bases de dados Lilacs, MedLine e Scielo. Foram incluídos estudos transversais ou longitudinais em inglês, português ou espanhol sem restrição de período de publicação que tinham como objetivo avaliar os níveis de estresse em profissionais de saúde que trabalham em serviços de emergência médica. Logo, nove artigos transversais e dois longitudinais foram incluídos, totalizando onze artigos. Resultados: Como resultado, observou-se que uma série de fatores ambientais (más condições de trabalho, atividades de administração) e pessoais (união estável, menor tempo de serviço) são associados ao estresse laboral. Todavia, principalmente os fatores relacionados ao ambiente são passíveis de intervenção. Conclusões: Dessa forma, surge a necessidade de políticas de melhoria do ambiente de trabalho e de empoderamento do profissional de saúde que trabalha em serviços de emergência médica.

Palavras-Chave: estresse ocupacional, serviços médicos de emergência, profissionais de saúde.

\section{ABSTRACT}

Introduction: The emergency medical services require the health professional to respond promptly to the demands of each patient. In this context, work stress can be extremely destructive not only for the worker, but also for the health service provided. Objective: Thus, this study aims to analyze the factors that trigger work stress in health professionals working in emergency medical services. Methods: For this, a integrative review of the literature was performed, based on the Lilacs, MedLine and Scielo databases. We included cross-sectional or longitudinal studies in English, Portuguese or Spanish without restriction of publication period that aimed to evaluate stress levels in health professionals working in emergency medical services. Therefore, nine transverse and two longitudinal articles were included, totaling eleven articles. Results: As a result, it was observed that a series of environmental factors (poor working conditions, administration activities) and personal factors (stable union, shorter working time) are associated with work stress. However, mainly environmental factors are amenable to intervention. Conclusions: Thus, there is a need for policies to improve the work environment and the empowerment of the health professional working in emergency medical services.

eywords: occupational stress, emergency medical services, health ofessionals. trodução 
O estresse é definido como o estado de resposta do indivíduo frente a um estímulo que provoque excitação emocional. Essa resposta ao fator estressor depende de características particulares de cada pessoa e da demanda do meio externo sobre ela. Quando essa discrepância é frequente, duradoura ou intensa, pode precipitar um esgotamento dos recursos do sujeito. Dessa forma, não ocorre uma perfeita adaptação do indivíduo, gerando manifestações clínicas diversas, que vão desde sintomas inespecíficos de depressão ou ansiedade a transtornos psiquiátricos bem definidos, como o transtorno de estresse pós-traumático ${ }^{1}$.

Essas manifestações são de extrema relevância ao serem analisadas individualmente; todavia, podem se tornar ainda mais importantes dependendo do meio no qual o indivíduo atua. Os serviços de emergência médica são complexos ao exigirem do profissional de saúde autocontrole e vasto conhecimento científico e humanescente para viabilizar uma pronta resposta às demandas particulares de cada paciente em um ambiente, muitas vezes, não favorável. Portanto, um profissional de saúde estressado poderia implicar em riscos para sua saúde, bem como para a segurança dos pacientes ${ }^{2}$. Um exemplo disso é que enfermeiros com 12 horas ou mais de trabalho apresentam três vezes mais chances de cometer um erro em comparação àqueles que desenvolvem suas atividades em turnos de 8,5 horas por $\mathrm{dia}^{3}$. Logo, o ambiente de trabalho pode influenciar negativamente a qualidade da assistência prestada.

Não obstante, é necessário apreender melhor o estresse e seus fatores desencadeantes no contexto dos serviços de emergência médica para vislumbrar possíveis avanços nas condições laborais desses serviços. Um ambiente de trabalho salutar e a saúde mental dos profissionais são fatores essenciais para uma qualificação contínua do cuidado à saúde, sobretudo nas situações de emergência médica ${ }^{4-5}$. Por isto, o objetivo deste estudo é analisar os fatores desencadeantes do estresse em profissionais da saúde que atuam em serviços de mergência médica.

\section{étodos}


O estudo consiste em uma revisão integrativa de literatura, que tem a finalidade de reunir e sintetizar resultados de pesquisas sobre um delimitado tema ou questão, de maneira sistemática e ordenada, contribuindo para o aprofundamento do conhecimento do tema investigado ${ }^{6}$.

A busca dos artigos científicos foi realizada nos meses de Agosto a Outubro de 2018, sendo utilizadas as bases de dados eletrônicas Lilacs, MedLine e Scielo. Como critérios de inclusão, os artigos deveriam ser estudos transversais ou longitudinais com o objetivo de avaliar o nível de estresse em profissionais da saúde atuantes em serviços de emergência médica; além disso, deveriam ter como idiomas de publicação português, inglês ou espanhol. Foram excluídos do estudo artigos que mensuravam o estresse por avaliações bioquímicas (nível de cortisol e de $\operatorname{Ig} \mathrm{A}$ ), artigos com o objetivo de avaliar a presença de doenças ou síndromes psiquiátricas bem definidas relacionadas ao estresse laboral e aqueles não disponíveis na íntegra na internet, bem como dissertações, teses e monografias.

As estratégias de busca utilizadas em cada base de pesquisa, assim como o número total de artigos obtidos e aqueles incluídos no estudo estão representados na figura 1.

Figura 1 - Processo de seleção dos artigos científicos
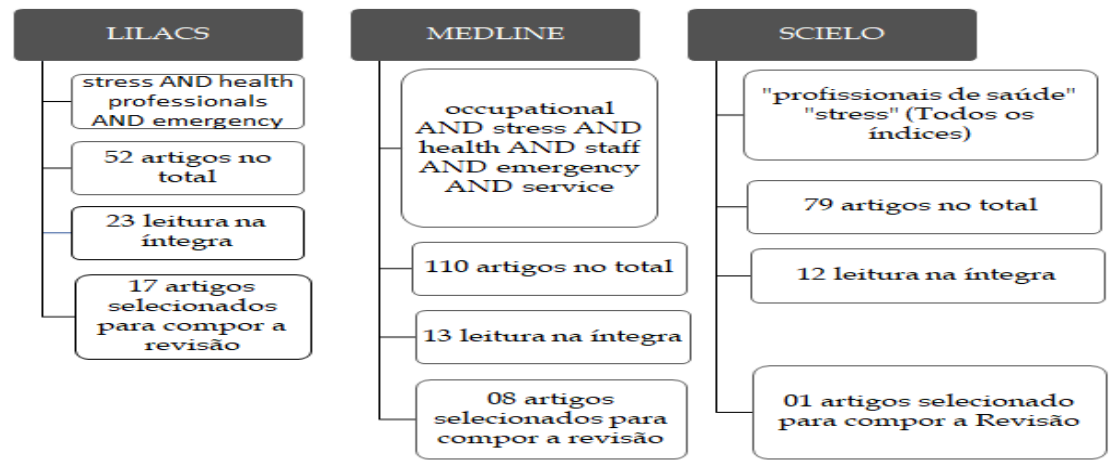

\section{esultados}

No total, 26 artigos científicos preencheram os critérios de inclusão e clusão preestabelecidos, sendo, por isso, incluídos no estudo. A tabela 1 
mostra um resumo dos pontos mais relevantes abordados nos trabalhos, assim como sua identificação.

Tabela 1 - Artigos incluídos no estudo

\begin{tabular}{|c|c|c|c|c|}
\hline \multicolumn{2}{|c|}{ Identificação } & \multicolumn{2}{|c|}{ Metodologia } & \multirow{2}{*}{ Achados } \\
\hline Autores & Ano & Desenho & Profissionais & \\
\hline $\begin{array}{l}\text { Theme Filha } \\
\text { MM, Costa } \\
\text { MAdS, } \\
\text { Guilam } \\
\text { MCR. }{ }^{7}\end{array}$ & 2013 & Transversal & $\begin{array}{l}\text { Assistentes de } \\
\text { enfermagem, } \\
\text { enfermeiros e } \\
\text { técnicos de } \\
\text { enfermagem }\end{array}$ & $\begin{array}{l}\text { Controle baixo, aliado com baixa } \\
\text { demanda (trabalho passivo) } \\
\text { prevaleceram, sendo contribuintes } \\
\text { para a insatisfação profissional e } \\
\text { autoavaliação negativa da saúde. } \\
\text { Alta tensão relacionou-se a menos } \\
\text { tempo na instituição, turnos à noite, } \\
\text { horas extra de trabalho, insatisfação } \\
\text { com o trabalho e baixo apoio social. }\end{array}$ \\
\hline $\begin{array}{l}\text { Sanchez Vera } \\
\text { KM, } \\
\text { Sandoval } \\
\text { Vegas MH, } \\
\text { Loli Ponce } \\
\text { RA. }{ }^{8}\end{array}$ & 2015 & Transversal & $\begin{array}{l}\text { Médicos, } \\
\text { enfermeiros } \\
\text { pilotos }\end{array}$ & $\begin{array}{l}\text { A prevalência de estresse } \\
\text { relacionado com o trabalho é alta } \\
\text { nos profissionais do Serviço Móvel } \\
\text { de Urgência (sem diferenças } \\
\text { significativas entre as profissões), } \\
\text { sendo relacionada à idade menor } \\
\text { que } 30 \text { anos. O nível de estresse no } \\
\text { trabalho foi inferior naqueles em } \\
\text { união estável e nos com mais de } 20 \\
\text { anos de serviço. }\end{array}$ \\
\hline
\end{tabular}

Menzani G, 2009 Transversal Enfermeiros Bianchi ERF. ${ }^{9}$
As áreas de maior nível de estresse foram as relacionadas às condições de trabalho, à administração pessoal (realizar tarefas com tempo mínimo disponível, elaborar escala mensal de funcionários, realizar atividades burocráticas) e coordenação das atividades da unidade. $\mathrm{O}$ escore médio de estresse por área foi de nível médio; todavia, esses profissionais estão sujeitos a atividades avaliadas como estressores fortes, como realizar atendimento aos familiares de pacientes críticos e realizar atendimento com tempo mínimo. 


\begin{tabular}{|c|c|c|c|c|}
\hline $\begin{array}{l}\text { Urbanetto } \\
\text { JdS, Silva } \\
\text { PCd, } \\
\text { Hoffmeister } \\
\text { E, Figueiredo } \\
\text { CEPd, Negri } \\
\text { BSd, Costa } \\
\text { BEPd.10 }\end{array}$ & 2011 & Transversal & $\begin{array}{lr}\text { Enfermeiros } & \mathrm{e} \\
\text { técnicos de } & \mathrm{de} \\
\text { enfermagem }\end{array}$ & $\begin{array}{l}\text { Baixo controle e baixa demanda } \\
\text { (trabalho passivo) prevaleceram. } \\
\text { Profissionais graduados foram } \\
\text { associados com o trabalho ativo e } \\
\text { baixa tensão; já profissionais sem } \\
\text { graduação, com trabalho passivo e } \\
\text { alta tensão. }\end{array}$ \\
\hline $\begin{array}{ll}\text { Aisling } & \mathrm{M}, \\
\text { Aisling } & \mathrm{D}, \\
\text { David C. } & \end{array}$ & 2016 & Transversal & $\begin{array}{l}\text { Profissionais da } \\
\text { ambulância, } \\
\text { enfermeiros, } \\
\text { médicos e } \\
\text { auxiliares de } \\
\text { apoio }\end{array}$ & $\begin{array}{l}\text { Níveis elevados de estresse } \\
\text { psicológico em cada profissão, } \\
\text { exceto em auxiliares de } \\
\text { ambulância. Correlação inversa } \\
\text { entre satisfação no trabalho e } \\
\text { estresse. Níveis elevados de } \\
\text { sintomatologia secundária a } \\
\text { trauma psicológico, sendo os níveis } \\
\text { mais altos em enfermeiros e } \\
\text { médicos. }\end{array}$ \\
\hline $\begin{array}{l}\text { Wu H, Sun } \\
\text { W, Wang L. }{ }^{11}\end{array}$ & 2012 & Transversal & Enfermeiras & $\begin{array}{l}\text { Sobrecarga, desbalanço entre } \\
\text { habilidades e função e conflito } \\
\text { entre a demanda de supervisores e } \\
\text { grupos de colegas de trabalho } \\
\text { tiveram maior associação com o } \\
\text { estresse ocupacional. }\end{array}$ \\
\hline $\begin{array}{l}\text { McPherson S, } \\
\text { Hale R, } \\
\text { Richardson } \mathrm{P}, \\
\text { Obholzer A. }{ }^{22}\end{array}$ & 2003 & Transversal & Médicos & $\begin{array}{l}\text { Níveis mais altos de estresse } \\
\text { psicológico entre médicos que } \\
\text { trabalham em serviços de acidente } \\
\text { e emergência do que entre outros } \\
\text { grupos de médicos. O } \\
\text { enfrentamento ativo (resolução de } \\
\text { problemas, planejamento, } \\
\text { reestruturação positiva) foi } \\
\text { negativamente ligado à ansiedade e } \\
\text { às queixas somáticas e foi } \\
\text { positivamente relacionado aos anos } \\
\text { decorridos desde a qualificação. }\end{array}$ \\
\hline $\begin{array}{l}\text { James AE, } \\
\text { Wright PL. }{ }^{13}\end{array}$ & 1991 & Transversal & $\begin{array}{l}\text { Profissionais do } \\
\text { serviço de } \\
\text { ambulância } \\
\text { (não especifica) }\end{array}$ & $\begin{array}{l}\text { As principais fontes de estresse } \\
\text { estão relacionadas com as relações } \\
\text { interpessoais (superiores, colegas, } \\
\text { funcionários do hospital, médicos). }\end{array}$ \\
\hline $\begin{array}{l}\text { D-M, Sun } \\
\text { Hong S, } \\
\text { Y-y, }\end{array}$ & 2015 & Transversal & Enfermeiros & $\begin{array}{l}\text { Os fatores estressores foram } \\
\text { principalmente relacionados à } \\
\text { especialidade de enfermagem e à }\end{array}$ \\
\hline
\end{tabular}

Urbanetto 2011 Transversal Enfermeiros e Baixo controle e baixa demanda JdS, Silva PCd, enfermagem Profissionais graduados foram associados com o trabalho ativo e baixa tensão; já profissionais sem graduação, com trabalho passivo e alta tensão.

BEPd.10

Wu H, Sun 2012 Transversal Enfermeiras

Sobrecarga, desbalanço entre entre a demanda de supervisores e grupos de colegas de trabalho tiveram maior associação com o

McPherson S, 2003 Transversal Médicos

Níveis mais altos de estresse psicológico entre médicos que trabalham em serviços de acidente emergência do que entre outros grupos de médicos. $\mathrm{O}$ enfrentamento ativo (resolução de planejamento reestruturação negativamente ligado à ansiedade e

James AE, 1991 Transversal Profissionais do As principais fontes de estresse Wright PL. ${ }^{13}$ ambulância Os fatores estressores foram principalmente relacionados à especialidade de enfermagem e à

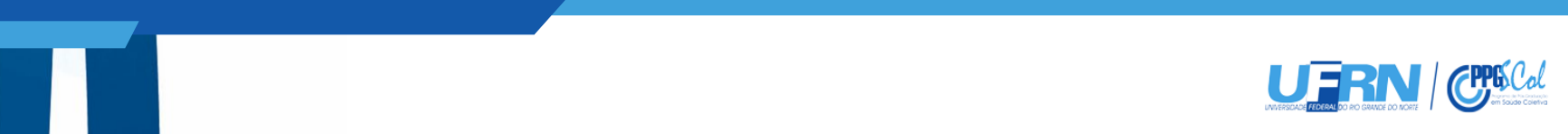


Kong F-y, Li

Q-j. ${ }^{14}$ grande carga de trabalho, incluindo baixos salários e benefícios, trabalho por turnos, pouca promoção e oportunidades, medo de cometer erros, acidentes relacionados ao trabalho, carga de trabalho pesada e falta de profissionais.

\section{Adriaenssens 2015 Longitudinal Enfermeiros} J, De Gucht V, Maes S. ${ }^{4}$

Maior percepção positiva da demanda, controle e apoio social no trabalho se correlacionaram com um aumento na satisfação no trabalho. Uma percepção mais positiva do controle e recompensa no trabalho foram associados com aumento no engajamento. Uma percepção mais positiva de demanda de trabalho e apoio social ao longo do tempo foram relacionados com os níveis mais baixos de exaustão emocional.

Revicki DA, 1996 Longitudinal Técnicos Gershon medicina

de Comportamento supervisionado, clareza de função e suporte de trabalho em grupo foram associados negativamente com estresse ocupacional e esse último foi associado a maior estresse psicológico.

A maioria dos profissionais com estresse laboral trabalha mais de dois fins de semana por mês, ficam nutricionistas, regularmente de plantão, trabalham
assistentes de em ambientes disfuncionais e não saúde participaram de reuniões de lazer regularmente. Dessa forma, os hospitais devem organizar horários com melhor balanço entre horas trabalhadas e não trabalhadas.

G, Gionta G,

Senergue $\mathrm{J}$,

Bèque

Lehucher-

Michel MP 16.
2015 Transversal

Enfermeiros, médicos, fisioterapeutas, nutricionistas,

Nas entrevistas realizadas, os do tipo estudo de caso
Enfermeiro, técnico enfermagem e auxiliar enfermagem do de fatores geradores de sofrimento no

e trabalho foram divididos em três

de subcategorias: impotência, dependência da atuação médica; 


\begin{tabular}{|c|c|c|c|}
\hline $\begin{array}{l}\text { g J, Vargas R, } \\
\text { Busnello GF } 17\end{array}$ & & $\begin{array}{lr}\text { setor de } & \text { Urgência } \\
\text { Emergência de } \\
\text { um hospital } \\
\text { localizado no } \\
\text { oeste de Santa } \\
\text { Catarina }\end{array}$ & $\begin{array}{l}\text { pouco reconhecimento do trabalho; } \\
\text { e sobrecarga de trabalho e pressão } \\
\text { psicológica. }\end{array}$ \\
\hline $\begin{array}{l}\text { Angelim } \\
\text { RCM, Rocha } \\
\text { GSA }^{18} \text {. }\end{array}$ & $\begin{array}{c}\text { Revisão } \\
\text { Integrativa }\end{array}$ & $\begin{array}{lr}\text { Produções } & \\
\text { sobre } & \text { as } \\
\text { condições de } & \text { drabalho do } \\
\text { pessoal de } & \text { de } \\
\text { enfermagem no } & \text { setor } \\
\text { urgência de } & \text { e } \\
\text { emergência, } & \\
\text { identificando se } \\
\text { esses fatores } \\
\text { têm gerado } \\
\text { desgaste e riscos } \\
\text { aos } \\
\text { trabalhadores }\end{array}$ & $\begin{array}{l}\text { A carga horária exaustiva e a baixa } \\
\text { remuneração são fatores } \\
\text { responsáveis pelo desgaste de } \\
\text { trabalho, tendo em vista que o } \\
\text { profissional acaba buscando vários } \\
\text { vínculos para poder obter uma } \\
\text { renda mensal satisfatória. A } \\
\text { insuficiência de recursos humanos } \\
\text { e materiais, ambiguidade de papéis } \\
\text { (acúmulo de funções) e violência } \\
\text { foram identificados como fatores } \\
\text { estressores. } \\
\text { Profissionais casados e com menos } \\
\text { tempo de serviço apresentaram } \\
\text { menor probabilidade ao a } \\
\text { adoecimento. }\end{array}$ \\
\hline $\begin{array}{l}\text { Rosado IVM, } \\
\text { Russo GHA, } \\
\text { Maia EMC }{ }^{19}\end{array}$ & Transversal & $\begin{array}{l}\text { Profissionais da } \\
\text { Medicina, } \\
\text { Enfermagem, } \\
\text { Serviço Social, } \\
\text { Odontologia, } \\
\text { Psicologia, } \\
\text { Fisioterapia, } \\
\text { Fonoaudiologia, } \\
\text { Nutrição e } \\
\text { Terapia } \\
\text { Ocupacional } \\
\text { atuantes em } \\
\text { dois Hospitais } \\
\text { de urgência e } \\
\text { emergência. }\end{array}$ & $\begin{array}{l}\text { Trabalho como fonte de desgaste da } \\
\text { saúde, em função de atributos } \\
\text { inerentes à própria atividade } \\
\text { profissional e ao ambiente em que } \\
\text { ela se realiza. } \\
\text { Fatores relacionados à função } \\
\text { desempenhada: esforço físico, } \\
\text { procedimentos repetitivos e } \\
\text { extenuante permanência em pé, } \\
\text { condições não ergonômicas nos } \\
\text { serviços investigados, } \\
\text { indisponibilidade de tempo para } \\
\text { descanso, lazer, atividades físicas, } \\
\text { práticas alimentares saudáveis, } \\
\text { convívio social e familiar, além de } \\
\text { outros cuidados com a própria } \\
\text { saúde. andores relacionados ao ambiente: } \\
\text { Fatores } \\
\text { Ambiente insalubre, turnos } \\
\text { prolongados, expedientes }\end{array}$ \\
\hline
\end{tabular}




\section{ciênncia
pural}

noturnos, sobrecarga de trabalho, precárias condições de trabalho, inexistência de planejamento ergonômico, relações autoritárias, inadequações na estrutura física, baixos salários.

Kogien M, 2014 Transversal Profissionais de Baixo discernimento intelectual, Cedaro JJ ${ }^{20} \quad$ enfermagem de baixo suporte social e vivenciar um um prontosocorro público estadual trabalho de alta exigência ou trabalho passivo (inércia motivacional em relação ao seu trabalho) foram os principais fatores de risco para prejuízos no domínio físico de qualidade de vida.

\begin{tabular}{lrc}
\hline Sobral PHAF, & 2013 & Revisão \\
Silva AMP, & & sistemática \\
Santos VEP, & & \\
Santos RAA, & & \\
Santos ALS &
\end{tabular}

Enfermeiros atuantes unidades urgência emergência
Ambulatorialização das unidades em de urgência e emergência, leva à de perda da identidade dessas e unidades e colabora para ineficiência do atendimento relacionada a superlotações majoritariamente com pacientes que podem ser atendidos em ambulatórios. Essa alteração da função das unidades de urgência e emergência associadas às condições de trabalho insalubres e inseguras com o cumprimento de tarefas prioritariamente burocráticas, carga de trabalho, a falta de profissionais qualificados na equipe de enfermagem, o ambiente físico impróprio e as características complexas dos serviços de emergência, levam o enfermeiro emergencialista a situações de estresse emocional o que contribui para a diminuição da qualidade de assistência desse profissional e de sua equipe como um todo. $\begin{array}{llllll}\text { Bezerra } & \text { FN, } 2012 & \begin{array}{c}\text { Revisão } \\ \text { integrativa }\end{array} & \begin{array}{l}\text { Enfermeiros } \\ \text { atuantes }\end{array} & \begin{array}{l}\text { Os resultados apontaram que o } \\ \text { estresse ocupacional dos }\end{array}\end{array}$ $\mathrm{VP}^{22}$ de enfermeiros de urgência

e




\section{ciência
pural}

urgência

emergência

e

escassez de recursos humanos e à
carga horária de trabalho, carga horária de trabalho,
instalações físicas e recursos materiais inadequados, além de plantões noturnos, interface trabalho-lar, relacionamentos interpessoais, trabalho em clima de competitividade e distanciamento entre teoria e prática. O sentido do trabalho para os profissionais contribui para sua proteção contra o sofrimento e o estresse ocupacional. Sugere-se que as instituições de saúde criem momentos e ambientes para que os profissionais compartilhem experiências e sentimentos vivenciados durante os plantões.

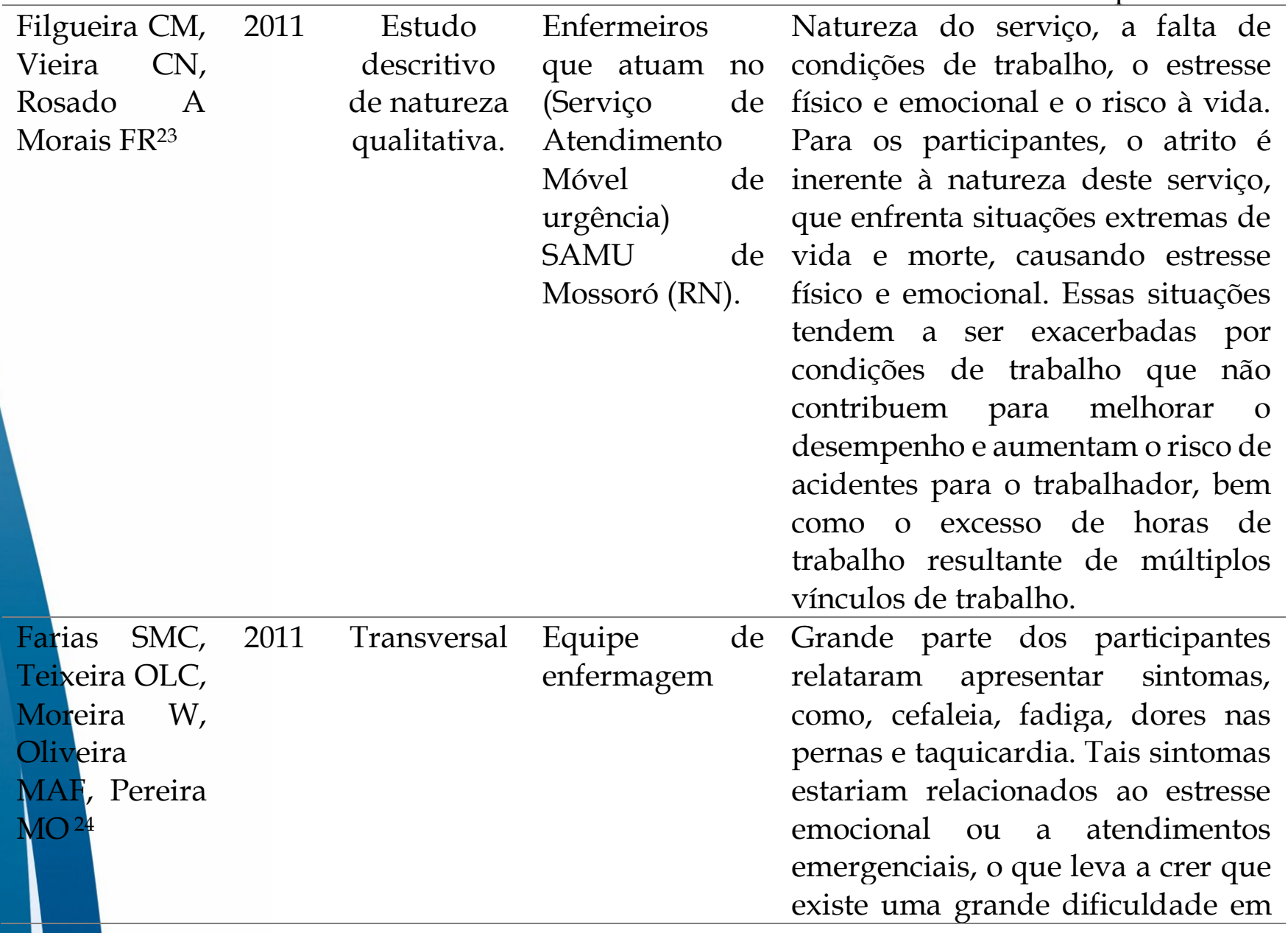




\begin{tabular}{|c|c|c|c|}
\hline & & & $\begin{array}{l}\text { se separar o estresse físico do } \\
\text { psíquico. }\end{array}$ \\
\hline $\begin{array}{l}\text { Dalri RCMB, } 2010 \\
\text { Robazzi } \\
\text { MLCC, Silva } \\
\text { LA. }{ }^{25}\end{array}$ & Transversal & $\begin{array}{l}\text { Equipe de } \\
\text { enfermagem }\end{array}$ & $\begin{array}{l}\text { Os trabalhadores de saúde da } \\
\text { emergência são sujeito a vários } \\
\text { riscos ocupacionais e, como } \\
\text { consequência deles, podem } \\
\text { desenvolver alterações de saúde } \\
\text { como algias, cansaço mental e } \\
\text { estresse, distúrbios do sono, } \\
\text { alterações cardiovasculares e } \\
\text { processos infecciosos. }\end{array}$ \\
\hline $\begin{array}{l}\text { Salomé GM, } 2009 \\
\text { Martins } \\
\text { MFMS, } \\
\text { Espósito } \\
\text { VHC. }{ }^{26}\end{array}$ & Transversal & $\begin{array}{l}\text { Equipe de } \\
\text { enfermagem }\end{array}$ & $\begin{array}{l}\text { Os profissionais de enfermagem } \\
\text { que trabalham na emergência } \\
\text { vivenciam momentos de estresse, } \\
\text { cansaço, esgotamento e frustração. } \\
\text { Os fatores que geram esses } \\
\text { sentimentos são: acúmulo de } \\
\text { funções, atividades burocráticas e } \\
\text { assistenciais e a limitação de tempo. }\end{array}$ \\
\hline $\begin{array}{l}\text { Aparecida CJ, } \\
\text { Dalri MCB, } \\
\text { Cyrillo RMS; } \\
\text { Saeki T, Veiga } \\
\text { RV }{ }^{27}\end{array}$ & Transversal & $\begin{array}{l}\text { Enfermeiros, } \\
\text { médicos, } \\
\text { condutores de } \\
\text { ambulância }\end{array}$ & $\begin{array}{l}\text { A equipe multiprofissional } \\
\text { vivencia a possibilidade de } \\
\text { sofrimento psíquico resultante de } \\
\text { fatores estressores inerentes a } \\
\text { situações de emergência. São } \\
\text { alguns deles: necessidade de } \\
\text { constante de capacitação técnica, } \\
\text { habilidade e agilidade, dificuldade } \\
\text { em lidar com sofrimento dos } \\
\text { familiares, trabalhar em locais } \\
\text { desfavoráveis, dificuldades em } \\
\text { lidar com a morte, impotência, } \\
\text { frustração e tristeza. }\end{array}$ \\
\hline $\begin{array}{l}\text { Almeida PJS, } \\
\text { Pires DEP28 }\end{array}$ & Transversal & $\begin{array}{l}\text { Médicos, equipe } \\
\text { de enfermagem, } \\
\text { assistente social }\end{array}$ & $\begin{array}{l}\text { Os serviços de emergência } \\
\text { apresentam vários estressores } \\
\text { como grande demanda, alta carga } \\
\text { de trabalho e estrutura física } \\
\text { inadequada. Por outro lado, foram } \\
\text { apontadas como fontes de prazer a } \\
\text { possibilidade de ajudar os usuários, } \\
\text { a possibilidade de exercer a } \\
\text { profissão na plenitude, a dinâmica } \\
\text { do serviço e a pouca existência de } \\
\text { rotinas. }\end{array}$ \\
\hline
\end{tabular}




\begin{tabular}{|c|c|c|c|c|}
\hline $\begin{array}{l}\text { Avellar LZ, } \\
\text { Valverde } \\
\text { PF. }^{29}\end{array}$ & 2007 & transversal & $\begin{array}{l}\text { Técnicos de } \\
\text { enfermagem }\end{array}$ & $\begin{array}{l}\text { O trabalho na emergência pode } \\
\text { apresentar várias adversidades, } \\
\text { como, grande demanda, má relação } \\
\text { com equipe de trabalho, vivências } \\
\text { de morte, necessidade de ações } \\
\text { rápidas. Isso gera sentimentos de } \\
\text { estresse, impotência, tristeza e } \\
\text { despersonalização } \\
\text { profissionais de saúde. }\end{array}$ \\
\hline $\begin{array}{l}\text { Fachini JS, } \\
\text { Scrigni AV, } \\
\text { Lima RCGS }\end{array}$ & 2017 & $\begin{array}{l}\text { Transversal } \\
\text { (estudo de } \\
\text { caso único) }\end{array}$ & $\begin{array}{l}\text { Trabalhadores } \\
\text { de equipe } \\
\text { multidisciplinar } \\
\text { de UTI } \\
\text { pediátrica } \\
\text { hospitalar (sete } \\
\text { médicos, cinco } \\
\text { enfermeiros, } \\
\text { dois fisioterapeutas, } \\
\text { um psicólogo, } \\
\text { um assistente } \\
\text { social, um } \\
\text { fonoaudiólogo e } \\
\text { um nutricionista). }\end{array}$ & $\begin{array}{l}\text { Sofrimento moral: gerado pela } \\
\text { incapacidade de agir, no trabalho, } \\
\text { com a necessária liberdade para } \\
\text { fazer escolhas autônomas, e pode } \\
\text { estar associado a conflitos éticos. } \\
\text { Provoca danos na vida pessoal, } \\
\text { trabalho e saúde. } \\
\text { Fatores relacionados ao sofrimento } \\
\text { moral: à limitação do acesso a } \\
\text { recursos tecnológicos e a } \\
\text { dificuldades para desenvolver e } \\
\text { integrar processo de trabalho } \\
\text { humanizado em saúde. Efeito } \\
\text { cascata: falta de recursos leva o } \\
\text { profissional a sofrimento moral, } \\
\text { rotinas levam a mecanização do } \\
\text { trabalho que, somada a múltiplos } \\
\text { empregos, propicia a } \\
\text { despersonalização, que também o } \\
\text { levará ao sofrimento. }\end{array}$ \\
\hline
\end{tabular}

Assim, analisando os dados da tabela, percebe-se que os fatores relacionados ao estresse em profissionais de saúde que trabalham em serviços de emergência médica podem ser divididos em dois principais grupos: os relacionados ao trabalho e aqueles relacionados ao trabalhador. No primeiro, tem-se idade menor que 30 anos e não estar em relação estável ${ }^{8}$ e, no segundo, más condições de trabalho ${ }^{9,11,14,15}$ e administração do serviço ${ }^{9}$. A figura 2 traz um esumo dos principais grupos de fatores segundo essa classificação.

Figura 2 - Grupos de fatores relacionados ao estresse em profissionais de saúde que trabalham em serviços de emergência médica 


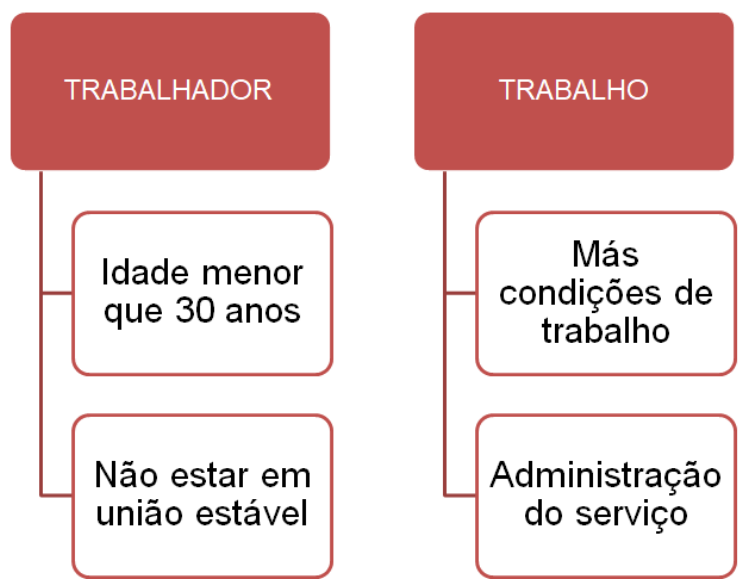

\section{Discussão}

Há um predomínio do trabalho passivo (baixa demanda e baixo controle) nos serviços de emergência médica, sendo essa categoria tão danosa quanto a de alta tensão (alta demanda e baixo controle). Isso se dá porque o desequilíbrio entre a demanda psicológica e o controle sobre o processo de trabalho resulta em tensão e perda de habilidades e interesse, resultando, assim, em uma menor autonomia do profissional. Logo, a necessidade de pensar antes de executar ações, mesmo que já rotineiras, pressupõem uma certa autonomia e proatividade profissional. Assim, a passividade laboral tenderia a reduzir a proatividade do profissional e, portanto, seu estímulo à efetividade do cuidado 7,10 .

Além disso, a maioria dos estressores foi encontrada no próprio ambiente de trabalho, sendo os principais pontos destacados: más condições de trabalho, atribuições relacionadas à administração do serviço e relações interpessoais conflituosas $^{9,13}$. As más condições de trabalho mais destacadas foram baixo salário, número inadequado de profissionais, carga de trabalho pesada, trabalho por turnos e pouca promoção e oportunidades ${ }^{11,18}$. Tais condições são indicativas de falta de incentivos e pouco reconhecimento para motivar os profissionais de aúde. Demonstram, portanto, a necessidade de planos de carreira e melhoria do hbiente de trabalho para motivá-los, além do estímulo ao processo de educação ntinuada. Ademais, atividades de administração do serviço (realizar tarefas 
com tempo mínimo disponível, elaborar escala mensal de funcionários, realizar atividades burocráticas) estão ligadas ao estresse à medida em que implicam em acúmulo de funções e a maior responsabilidade tanto no que diz respeito a recursos humanos, quanto materiais para o bom funcionamento do serviço. Tal função gera conflitos de valores pelo fato de envolver o interesse de várias pessoas e, com isso, a incumbência de direcionar para o objetivo comum é, por vezes, desgastante ${ }^{9,13}$.

Por outro lado, fatores relacionados ao próprio trabalhador também se associam à sua resposta frente aos estressores, tanto de forma negativa, quanto positiva. Foi observado que a presença de união estável é um fator protetor para o estresse laboral ${ }^{8,10}$. Esta pode ser vista como uma fonte de apoio psicossocial ao indivíduo, visto que pessoas com companheiros teriam alguém para compartilhar angústias e ter momentos de lazer e descontração pessoal de forma a aliviar o estresse ${ }^{8}$. Por sua vez, idade menor que 30 anos foi mais relacionada ao estresse. Isso acontece, porque o maior tempo de serviço estaria relacionado à naturalização dos fatores estressantes, assim como maior adaptação e preparo do indivíduo para manejá-los. A idade menor que trinta anos, seria uma variável proxy do menor tempo de serviço e, portanto, as pessoas ainda não estariam “adaptadas"8,10.

Como consequência do desequilíbrio de todos esses fatores, o estresse laboral resultante traz malefícios para o trabalhador, como insatisfação profissional, autoavaliações negativas da saúde e até sintomas emocionais, físicos e comportamentais ${ }^{5,7}$. Tal quadro no cenário de um serviço de emergência médica pode ser desastroso, tendo em vista a complexidade da assistência prestada nesse local, a qual exige rápida resposta dos profissionais ${ }^{2}$. Um estudo revelou que em trabalhadores atuantes por mais de 40 horas semanais, o risco para cometer incidentes aumentou em 46\%. Dessa forma, não há como separar sistência prestada e vivência de trabalho pelo profissional de saúde 3 . 
Em meio a tais achados, é importante atentar que o estudo apresenta limitações. Em relação à seleção dos artigos, foram incluídas apenas as línguas português, inglês e espanhol. Além disso, a grande heterogeneidade das metodologias dos estudos incluídos, seja em relação à amostra ou ferramenta de coleta, podem trazer dificuldades na interpretação dos resultados.

\section{Conclusão}

Portanto, deve-se atentar que um número considerável dos estressores presentes no trabalho é passível de intervenção. Logo, surge a necessidade de políticas de melhoria das condições de trabalho e de apoio psicológico aos profissionais de saúde que trabalham em serviços de emergência médica. Tais programas psicológicos devem objetivar empoderar o profissional para o enfrentamento ativo dos fatores estressores ao seu redor para que tenha mais controle sobre a situação. 


\section{Referências}

1. Margis R, Picon P, Cosner AF, Silveira RDO. Relação entre estressores, estresse e ansiedade. Rev psiquiatr Rio Gd Sul.2003; 25(supl.1):65-74.

2. Panizzon C, Luz AMH, Fensterseifer LM. Estresse da equipe de enfermagem de emergência clínica. Rev Gaucha Enferm. 2008; 29(3):391-9.

3. Caruso CC. Negative Impacts of Shiftwork and Long Work Hours. Rehabilitation nursing: the official journal of the Association of Rehabilitation Nurses. 2014;39(1):16-25.

4. Adriaenssens J, De Gucht V, Maes S. Causes and consequences of occupational stress in emergency nurses, a longitudinal study. J Nurs Manag. 2015;23(3):346-58.

5. Aisling M, Aisling D, David C. An Assessment of Psychological Need in Emergency Medical Staff in the Northern Health and Social Care Trust Area. Ulster Med J. 2016;85(2):92-8.

6. Souza MT, Silva MD, Carvalho R. Revisão integrativa: o que é e como fazer. Einstein. 2010; 8(1 Pt 1):102-6.

7. Theme Filha MM, Costa MAdS, Guilam MCR. Occupational stress and self-rated health among nurses. Rev Lat Am Enfermagem. 2013;21(2):475-83.

8. Sanchez Vera KM, Sandoval Vegas MH, Loli Ponce RA. Prevalencia de estrés laboral en el personal asistencial prehospitalario del programa de sistema de atención móvil de urgencias -Instituto de Gestión de Servicios de Salud. Rev enferm herediana. 2015;8(2):116-22.

Menzani G, Bianchi ERF. Stress dos enfermeiros de pronto socorro dos hospitais brasileiros. Rev eletrônica enferm. 2009;11(2):327-33. 
10. Urbanetto JdS, Silva PCd, Hoffmeister E, Figueiredo CEPd, Negri BSd, Costa BEPd. Workplace stress in nursing workers from an emergency hospital: Job Stress Scale analysis. Rev Lat Am Enfermagem. 2011;19(5):1122-31.

11. Wu H, Sun W, Wang L. Factors associated with occupational stress among Chinese female emergency nurses. Emerg Med J. 2012;29(7):554-8.

12. McPherson S, Hale R, Richardson P, Obholzer A. Stress and coping in accident and emergency senior house officers. Emerg Med J. 2003;20(3):230-1.

13. James AE, Wright PL. Occupational stress in the ambulance service. Health Manpow Manage. 1991;17(4):4-11.

14. Lu D-M, Sun N, Hong S, Fan Y-y, Kong F-y, Li Q-j. Occupational stress and coping strategies among emergency department nurses of China. Arch Psychiatr Nurs. 2015;29(4):208-12.

15. Revicki DA, Gershon RR. Work-related stress and psychological distress in emergency medical technicians. J Occup Health Psychol. 1996;1(4):391-6.

16. Bellagamba G, Gionta G, Senergue J, Bèque C, Lehucher-Michel MP. Organizational factors impactinig job strain and mental quality of life in emergency and critical care units. International Journal of Occupational Medicine and Environmental Health. 2015; 28(2): 357-367.

17. Kohls M, Olschowsky A, Barreta NL, Schimerfening J, Vargas R, Busnello GF. A enfermagem na urgência e emergência: entre o prazer e o sofrimento. Rev. pesqui. cuid. fundam. (Online). 2017; 9(2): 422-431.

18. Angelim RCM, Rocha GSA. Produção científica acerca das condições de trabalho da enfermagem em serviços de urgência e emergência. Rev. pesqui. cuid. fundam. (Online). 2016; 8(1): 3845-3859.

Rosado IVM, Russo GHA, Maia EMC. Produzir saúde suscita adoecimento? contradições do trabalho em hospitais públicos de urgência e emergência. nc. Saúde Cole. 2015;20(10): 3021-3032. 
20. Kogien M, Cedaro JJ. Pronto-socorro publico: impactos psicossociais no dominio fisico da qualidade de vida de profissionais de enfermagem. Rev Lat Am Enfermagem. 2014;22(1): 51-58.

21. Sobral PHAF, Silva AMP, Santos VEP, Santos RAA, Santos ALS. Atuação de enfermagem em serviços de emergência: revisão sistemática. Rev. pesqui. cuid. fundam. (Online). 2013; 5(4): 396-407.

22. Bezerra FN, Silva TM, Ramos VP. Estrés laboral de los enfermeros de urgencia y emergencia: revisión integradora de literatura. Acta paul. Enferm. 2012; 25(spe2): 151-156.

23. Filgueira CM, Vieira CN, Rosado A Morais FR. El desgaste relacionado con el trabajo desde la perspectiva de los enfermeros de atención pre-hospitalaria Rev Cubana Enferm. 2011; 27(4): 309-318.

24. Farias SMC, Teixeira OLC, Moreira W, Oliveira MAF, Pereira MO. Caracterização dos sintomas físicos de estresse na equipe de pronto atendimento. Rev Esc Enferm USP. 2011; 45(3): 722-729.

25. Dalri RCMB, Robazzi MLCC, Silva LA. Riscos ocupacionais e alterações de saúde entre trabalhadores de enfermagem brasileiros de unidades de urgência e emergência. Cienc. Enferm. 2010; 16(2): 69-81.

26. Salomé GM, Martins MFMS, Espósito VHC. Sentimentos vivenciados pelos profissionais de enfermagem que atuam em unidade de emergência. Rev Bras Enferm. 2009; 62(6): 856-862.

27. Aparecida CJ, Dalri MCB, Cyrillo RMS; Saeki T, Veiga RV. Vivências de uma equipe multiprofissional de atendimiento pre-hospitalar móvel em suporte avançado de vida na assistência ao adulto em situação de parada diorrespiratória. Cienc. Enferm. 2008; 14(2): 97-105. 


\section{ciênciucia
purl}

28. Almeida PJS, Pires DEP. O trabalho em emergência: entre o prazer e o sofrimento.Rev. eletrônica enferm. 2007; 9(3):617-629.

29. Avellar LZ, Valverde PF. Sofrimento psíquico em trabalhadores de enfermagem de uma unidade de oncologia. Psicol. Estud. 2007;12(3): 475-481.

30. Fachini JS, Scrigni AV, Lima RCGS .Sofrimento moral de trabalhadores de uma UTI pediátrica. Revista Bioética. 2017; 25(1): 111 - 122. 